J. Korean Math. Soc. 50 (2013), No. 2, pp. 425-444

http://dx.doi.org/10.4134/JKMS.2013.50.2.425

\title{
SOME HYPERBOLIC SPACE FORMS WITH FEW GENERATED FUNDAMENTAL GROUPS
}

\author{
Alberto Cavicchioli, Emil Molnár, And Agnese I. Telloni \\ ABStRaCt. We construct some hyperbolic hyperelliptic space forms wh- \\ ose fundamental groups are generated by only two or three isometries. \\ Each occurring group is obtained from a supergroup, which is an extended \\ Coxeter group generated by plane reflections and half-turns. Then we \\ describe covering properties and determine the isometry groups of the \\ constructed manifolds. Furthermore, we give an explicit construction of \\ space form of the second smallest volume nonorientable hyperbolic 3- \\ manifold with one cusp.
}

\section{Introduction}

A complete connected Riemannian $n$-manifold of constant sectional curvature, briefly called a space form, can be considered as an orbit space $X^{n} / G$, where $X^{n}$ is one of the classical spaces $\mathbb{S}^{n}, \mathbb{R}^{n}$, and $\mathbb{H}^{n}$ (spherical, Euclidean, and hyperbolic $n$-space, respectively) and $G$ is an isometry group acting discontinuously and freely on $X$. With regards to the general theory, we refer for example to the monograph [26] by J. A. Wolf. For hyperbolic space forms, see Burago's appendix to the Russian translation of the book, where basic results of G. D. Mostow, G. A. Margulis and W. P. Thurston are also presented. We refer also to [22] for a more recent monograph on the topic. A closed $n$-manifold $M^{n}$ is said to be hyperelliptic if it has an orientation-reversing involution $\tau$ such that the quotient space $M^{n} /\langle\tau\rangle$ is homeomorphic to the $n$-sphere $\mathbb{S}^{n}$. In this case, $\tau$ is called a hyperelliptic involution. If a manifold can be equipped with a geometric structure, then $\tau$ is assumed to be an isometry. Three-dimensional hyperelliptic manifolds are objects of special interest because of their relation with the Knot Theory. If $M^{3}$ is a hyperelliptic 3-manifold with a hyperelliptic involution $\tau$, then $M^{3}$ is the 2 -fold branched covering of the 3 -sphere $\mathbb{S}^{3}$ branched over a link (in particular, a knot) $L$. The covering is given by the action of $\tau$ and each point of $L$ has branching index 2. In other words,

Received June 26, 2012; Revised September 17, 2012.

2010 Mathematics Subject Classification. Primary 57M12, 57N10; Secondary 57M05, $57 \mathrm{M} 50$.

Key words and phrases. hyperbolic 3-manifold, hyperelliptic involution, cyclic branched covering, Heegaard diagram, hyperbolic orbifold, fundamental group, isometry group. 
$M^{3}$ is the 2 -fold covering of a $\pi$-orbifold $\mathcal{O}=\mathbb{S}^{3}(L)$ with underlying space $\mathbb{S}^{3}$ and singular set $L$ with singular angle $\pi$ at each point of $L$. In [14], it was shown that in any of the eight three-dimensional geometries, there exists a 3 manifold with a geometric hyperelliptic involution. If $M^{3}$ is a closed hyperbolic 3-manifold, then by Mostow's Rigidity Theorem, a hyperelliptic involution is equivalent to an isometric hyperelliptic involution. Even in the hyperbolic case a hyperelliptic involution is, in general, not unique. Many examples of hyperelliptic 3-manifolds (also admitting one, two, or three hyperelliptic involutions) were constructed by several authors (see for example [23] and its references). Notice that the structure and the fixed point set of a hyperelliptic involution is unknown, even in the simplest cases. In this paper, we construct some hyperelliptic hyperbolic 3 -manifolds to illustrate the difficulties in developing a classification of the fixed point loci for hyperelliptic transformations of such a class of manifolds. However, the main motivation of the paper is to describe a concrete geometric method for determining explicitly the branch set of a hyperelliptic involution from a combinatorial representation (as a polyhedral scheme or a Heegaard diagram) of the considered manifold. In fact, we combine techniques of algebraic and geometric topology to find an explicit form for such an involution in some interesting examples, giving a geometrical as well as an algebraic description of it. In more detail, we construct four examples of hyperbolic 3-space forms, denoted by $M_{2}, M, M_{5}$, and $M^{\prime}$. The manifolds $M_{5}$ and $M^{\prime}$ are hyperelliptic with 2-generated fundamental groups (see Section 5). They are obtained from Archimedian solids by pairwise identifications of the boundary faces. The manifold $M_{2}$ is hyperelliptic with 3-generated fundamental group (see Section 3). It is also a space form but its fundamental domain is "cubic". In Section 4, a non-compact hyperbolic space form of finite volume will be constructed which is non-orientable and its fundamental group has two generators. The first example of such a type was constructed by Gieseking (1912). In particular, we show that our example is precisely the second smallest volume nonorientable hyperbolic 3-manifold with one cusp. The unified method for constructing our hyperbolic space forms is as follows (this is also illustrated by a simpler case in Section 3): (1) starting with a hyperbolic Coxeter group $L$, take a finite index torsion-free subgroup $G$ of $L$ or an index two extension of $L ;(2)$ determine explicitly a fundamental polyhedron $\mathbb{P}_{G}$ for the $G$-action in the hyperbolic 3 -space $\mathbb{H}^{3}$ (this means that we have a more general situation than in the constructions known thus far $[12,16,19]) ;(3)$ construct from the polyhedron $\mathbb{P}_{G}$ (together with a pairwise identification of its boundary faces) a Heegaard diagram for the quotient space form $M=\mathbb{H}^{3} / G$. From a 2-symmetric Heegaard diagram, we describe $M$ as the 2 -fold covering of the 3-sphere branched over a well-specified 3-bridge link. Then we completely determine the isometry group of $M$. Nowadays, many compact hyperbolic $3-$ manifolds with two generators and - at the same time - of Heegaard genus two are known although complete classifications of neither are given (see e.g. [9] and $[10]$ ). However, the equivalence - between a manifold with a two-generator 
fundamental group and that of a possible Heegaard splitting of genus two, for compact hyperbolic 3-manifolds - is only a conjecture (not yet proved by our latest information). The basic classification problem of two-generator hyperbolic space forms is also still open. On the other hand, Heegaard splitting turns out to be the most appropriate technique for studying the topology of such space forms.

\section{Background on hyperellipticity and Heegaard diagrams}

To clarify the reading of the next sections, we briefly recall some definitions and results on hyperellipticity and on the combinatorial representation of closed connected 3-manifolds via Heegaard diagrams. For more details on hyperelliptic manifolds, see for example [7]. For basic facts and results on Heegaard diagrams and branched coverings, we refer among others to an excellent textbook on 3-manifold topology [8] (see also [3]).

2.1) Hyperellipticity. Let us consider the quadratic equation $y^{2}=p(x)$ for $(x, y) \in \mathbb{C}^{2}$, where $p(x)$ is a polynomial of even degree $2 g+2(g>0)$ whose coefficients are real numbers. The solution set (locus) of this equation defines a Riemann surface, which is a 2 -fold branched covering of $\mathbb{C}$. The cover is obtained by projection to the $x$-coordinate. The branching set corresponds to the roots of $p(x)$. There is a unique smooth closed Riemann surface $\Sigma_{g}$ of genus $g$ naturally associated with the above equation. Moreover, there is a holomorphic map from $\Sigma_{g}$ onto $\mathbb{C} \cup\{\infty\}=\mathbb{S}^{2}$ which extends the projection to the $x$-coordinate. All such surfaces are hyperelliptic by construction and vice versa, that is, any hyperelliptic closed orientable Riemann surface can be obtained in this way. The hyperelliptic involution $\tau$ of $\Sigma_{g}$ flips the two sheets of the double cover of $\mathbb{S}^{2}$ and has exactly $2 g+2$ fixed points, called the Weierstrass points of $\Sigma_{g}$. This involution is unique and lies in the center of the (finite) isometry group of $\Sigma_{g}$. Hyperelliptic surfaces are the simplest Riemann surfaces and have many interesting properties. For example, modular transformations can be generated by permutations of the branch points. This is significant in that it simplifies the construction of modular invariants. The dimension of this set of surfaces, as a subspace of moduli space, can be found as follows. Analytic mappings of the complex plane into itself (that is, elements of $\mathrm{SL}(2 ; \mathbb{C})$ ) can be used to fix three of the Weierstrass points. This leaves $(2 g-1)$ points, giving $(2 g-1)$ complex parameters. There are some common features between hyperelliptic Riemann surfaces and hyperelliptic 3-manifolds when considering the Heegaard genus (defined below) instead of the genus. However, in general, the situation for 3-manifolds is much more complicated. For example, hyperelliptic 3-manifolds may have an arbitrarily high number of nonconjugate hyperelliptic involutions. It is also known that a hyperelliptic involution of a closed hyperbolic 3-manifold $M^{3}$ with Heegaard genus 2 may not be in the center of the isometry group of $M^{3}$ (see [20], Theorem 1). Moreover, there is no explicit classification of the fixed point locus for hyperelliptic isometries of 
hyperbolic 3-manifolds. Finally, we observe that few results are known about the same problem for hyperelliptic manifolds in higher dimensions, some of them obtained only for the class of hyperelliptic Lefschetz fibrations.

2.2) Heegaard diagrams. A Heegaard splitting of a closed connected orientable 3-manifold $M$ is a pair $(V, W)$ of homeomorphic orientable compact cubes with handles such that $M=V \cup W$ and $V \cap W=\partial V=\partial W$. The closed connected orientable surface $F=\partial V=\partial W$ is called the Heegaard surface of the splitting $(V, W)$ of $M$. A classical theorem of Heegaard states that every closed connected orientable $3-$ manifold $M$ admits a Heegaard splitting. This splitting can be constructed as follows. Consider the 1-skeleton of a simplicial triangulation of $M$ and define $V$ as a regular neighbourhood of it. Then we set $W$ to be the closure of the complement of $V$ in $M$. The Heegaard genus $g(M)$ of $M$ is the smallest integer $g$ such that $M$ has a Heegaard surface of genus $g$. Given a splitting $(V, W)$ of $M$, let $D_{1}, \ldots, D_{g}$ be a collection of pairwise disjoint properly embedded discs in $W$ which cut $W$ into a 3-cell. The pairwise disjoint simple closed curves $\mathbf{w}_{i}=\partial D_{i}$ cut $F=\partial W$ into a 2-sphere with $2 g$ holes. We say that $\mathbf{w}=\left\{\mathbf{w}_{1}, \ldots, \mathbf{w}_{g}\right\}$ is a set of meridians of the handlebody $W$. Let $\mathbf{v}=\left\{\mathbf{v}_{1}, \ldots, \mathbf{v}_{g}\right\}$ be a set of meridians of the handlebody $V$. Then the triple $(F, \mathbf{v}, \mathbf{w})$ is called a Heegaard diagram associated to the splitting $(V, W)$ of $M$ (or briefly, a Heegaard diagram of $M$ ). The diagram can be drawn in a plane by flattening the above 2 -sphere with $2 g$ holes (whose quotient space is $F$ ). In this case, a set of meridians can be re-obtained by identifying in pairs the boundaries of the holes, while the other one gives rise to a set of pairwise disjoint simple arcs connecting the boundaries of the holes. The construction produces a planar graph (together with a pairing of the holes) which completely represents the manifold $M$ in the sense that $M$ can be recovered from it (for details, see for example [8], Chap. 5). Of course, there exist many different Heegaard diagrams representing the same manifold. The equivalence problem was solved by Singer: two different Heegaard diagrams of the same 3-manifold are related by a finite sequence of certain elementary moves (and/or their inverses), called Singer's moves. The first move changes the orientation on a curve of the diagram $(F, \mathbf{v}, \mathbf{w})$ or shifts a curve by isotopy. The second move substitutes a curve $\mathbf{w}_{i}$ of $\mathbf{w}$ with a curve $\mathbf{w}^{\prime}{ }_{i}$ after a slight shifting to make $\mathbf{w}_{i}^{\prime}$ disjoint from $\mathbf{w}_{k}$ for $i \neq k$. The curve $\mathbf{w}_{i}^{\prime}$ is obtained by a connected sum of the curves $\mathbf{w}_{i}$ and $\mathbf{w}_{k}$. This operation is defined similarly for the set of meridians $\mathbf{v}$. The last move adds a trivial (i.e., unknotted) handle and a trivial curve to the diagram. It follows that Heegaard diagrams (up to Singer's moves) give an adequate representation of the closed connected orientable 3 manifolds in the sense that all invariants of the represented manifolds can be obtained from their diagrams via graph-theoretical algorithms. An interesting open problem in the theory of Heegaard diagrams is the famous conjecture that the Heegaard genus of a closed connected hyperbolic 3-manifold is equal to the number of minimal generators for the fundamental group. Any closed orientable 3-manifold of Heegaard genus two admits an orientation preserving 
involution whose quotient space is $\mathbb{S}^{3}$ and whose branching locus is a 3-bridge link. Connections between Heegaard diagrams and Branched coverings can be found for example in $[3,21]$. In particular, it is known that every closed connected orientable 3 -manifold represented by a 2-symmetric Heegaard diagram is homeomorphic to the 2-fold covering of the 3-sphere branched over a link. Following [3], we recall that a Heegaard diagram associated to the splitting ( $V, W$ ) of $M$ is said to be 2-symmetric if it satisfies the following conditions: (1) there is an orientation-preserving involution $\rho$ of $M$ which sends $V$ onto $V$ (resp., $W$ onto $W$ ); (2) the orbit space $V / \rho$ (resp., $W / \rho$ ) of $V$ (resp., $W$ ) under the action of $\rho$ is a 3-ball; and (3) the image of the fixed point set of $\rho$ is an unknotted set of arcs in the ball $V / \rho$ (resp., $W / \rho)$. We expect that our methods and results may be helpful to determine explicitly the branching sets starting from a combinatorial representation of a hyperelliptic 3-manifold.
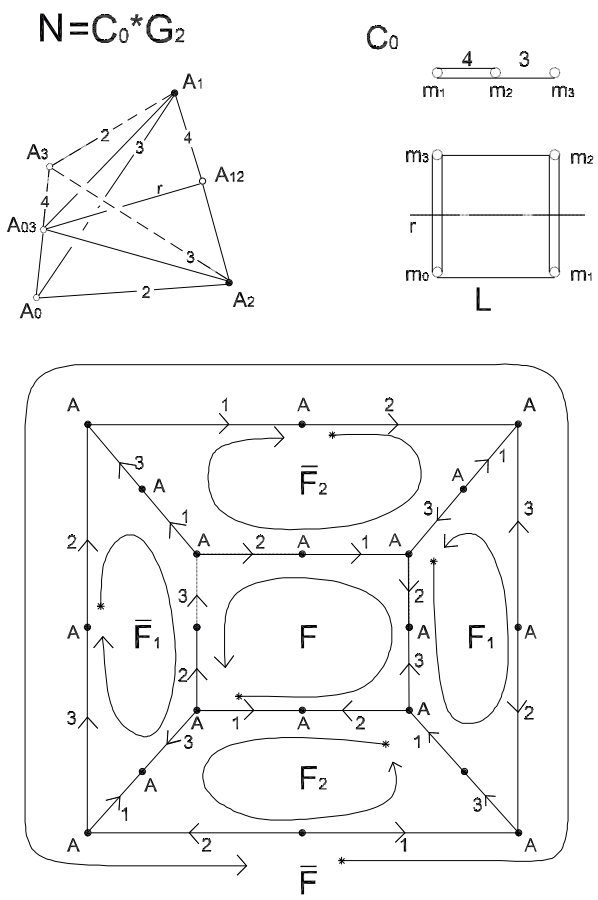

Figure 1. The hyperbolic manifold $M_{2}=\mathbb{H}^{3} / G_{2}$

\section{A space form with "cubic" fundamental domain}

Consider a Lanner group $L$ as a Coxeter group generated by reflections in the face planes of the tetrahedron $A_{0} A_{1} A_{2} A_{3}$ in Figure 1. The Coxeter diagram 
$[6,11]$ shows also the angular conditions of the mirror planes $m_{i}$ opposite from the vertices $A_{i}$. For instance, $m_{1}$ and $m_{2}$ have an angle $\pi / 4$, and $m_{0}$ and $m_{2}$ are perpendicular. This tetrahedron can be realized in $\mathbb{H}^{3}$. We introduce the half-turn $r$ which changes $m_{0}$ and $m_{1}$ to $m_{3}$ and $m_{2}$, respectively. So we get a group $N=\langle L, r\rangle$ as a semi-direct product. Now we get a fundamental domain $\mathcal{F}_{N}$ for $N$ by bisecting at edge $A_{1} A_{2}$ and taking the part with the vertex $A_{0}$. The stabilizer of $A_{0}$ in $N$, denoted by $C_{0}$, is just the complete symmetry group of a cube. Then $C_{0}$ is generated by reflections $m_{1}, m_{2}$ and $m_{3}$ (see Figure 1 ). We construct a new concave polyhedron $\mathbb{P}_{2}$ by uniting the $C_{0}$-images of $\mathcal{F}_{N}$ around $A_{0}$ as a center. Then $\mathbb{P}_{2}$ becomes a "cube" with six broken faces $F, \bar{F}$, $F_{1}, \bar{F}_{1}, F_{2}$, and $\bar{F}_{2}$ with eight "ordinary" vertices (i.e., the $C_{0}$-images of $A_{1}$ ) and twelve "additional" vertices in the middle of the broken edges (i.e., the $C_{0^{-}}$ images of $A_{2}$ ). The Schlegel diagram of $\mathbb{P}_{2}$ is described in Figure 1. The point is that $\mathbb{P}_{2}$ becomes a fundamental domain of the group $G_{2}$ to be constructed. We introduce appropriate screw motions $s, s_{1}, s_{2}$ as generators of $G_{2}$ pairing the faces of $\mathbb{P}_{2}$ with each other. In Figure 1 , these are indicated by corresponding arrows. The screw motion $s$ maps the face $F$ onto the face $\bar{F}$ and $\mathbb{P}_{2}$ onto its image $\mathbb{P}_{2}^{s}$ along the face $\bar{F}$; the inverse screw motion $s^{-1}$ acts analogously. The screw motion $s_{i}, i=1,2$, maps the face $F_{i}$ onto the face $\bar{F}_{i}$ according to the arrows in Figure 1. We have to guarantee the free action of $G_{2}$ so that the identified polyhedron $\mathbb{P}_{2}$ has only points with a ball-like neighbourhood. This is indeed the case. Let us list the following three arguments.

1) The two face domains around the midpoint $A_{03}$ and its $G_{2}$-equivalents fill a ball-like neighbourhood because the stabilizer $C_{03}$ of $A_{03}$ in $N$ is of order 16 and the group $C_{0} \cap C_{03}=\left\langle m_{1}, m_{2}\right\rangle$ has eight elements. There are three such equivalence classes.

2) The eight angular domains around the midpoint $A_{12}$ and its $G_{2}$-equivalents do the same since the stabilizer $C_{12}$ of $A_{12}$ in $N$ is of order 16 and $C_{0} \cap C_{12}=$ $\left\langle m_{3}\right\rangle$ has two elements. We have three such equivalence classes.

3) All the vertex domains belonging to the $G_{2}$-equivalence class of $A_{1} \sim A_{2}$, fill a ball-like neighbourhood again since the stabilizers $C_{1}$ of $A_{1}$ and $C_{2}$ of $A_{2}$, conjugated in the supergroup $N$ by $r$, have the same order as $C_{0}$.

We mention that each generator of $G_{2}$ can be expressed by those of the supergroup $N$. For instance, we have $s=r m_{3} m_{2} m_{1} m_{2} m_{3} m_{2}$. More importantly, for each edge equivalence class, we can write the corresponding cycle relation by the algorithm of Poincaré (see [13, 16, 17, 18, 19]):

$$
\begin{aligned}
& \text { edge class } 1: s_{2} s_{1} s_{2} s s_{2}^{-1} s_{1} s_{2}^{-1} s^{-1}=1 \\
& \text { edge class } 2: s s_{1}^{-1} s s_{2}^{-1} s s_{1} s s_{2}=1 \\
& \text { edge class } 3 \quad: \quad s_{1} s_{2} s_{1} s^{-1} s_{1}^{-1} s_{2} s_{1}^{-1} s=1
\end{aligned}
$$

Then $G_{2}$ is the group with generators $s, s_{1}, s_{2}$ with the previously defined relations. The polyhedral 3-cell $\mathbb{P}_{2}$ appears in the hyperbolic 3 -space $\mathbb{H}^{3}$ and its planar faces are paired by isometries that satisfy the above cyclic conditions. Then the Poincaré Polyhedron Theorem (see, for example, [13]) shows that $\mathbb{P}_{2}$ 
is a fundamental region for the discontinuous group of isometries $G_{2}$ generated by the identifications of the sides. So $G_{2}$ presents the hyperbolic compact space form $M_{2}=\mathbb{H}^{3} / G_{2}$ by the identified polyhedron $\mathbb{P}_{2}$. The number of generators of $G_{2}$ cannot be reduced, and we get $G_{2}^{a b}=H_{1}\left(M_{2} ; \mathbb{Z}\right) \cong \mathbb{Z}_{2} \oplus \mathbb{Z}_{2} \oplus \mathbb{Z}_{4}$ by abelianization to obtain the first integer homology group of $M_{2}$, as denoted before. Summarizing, the supergroup $N$ is decomposed in the form $N=C_{0} * G_{2}$ with $G_{2}$ acting freely on $\mathbb{H}^{3}$. This means that each element of $N$ can uniquely be written as $n=c_{0} * g_{2}, n \in N, c_{0} \in C_{0}$ and $g_{2} \in G_{2}$.

Theorem 3.1. Let $M_{2}=\mathbb{H}^{3} / G_{2}$ be the closed (compact and without boundary) hyperbolic 3-manifold constructed above. The finite presentation

$$
\begin{aligned}
G_{2}=\left\langle s, s_{i}(i=1,2) \quad: \quad\right. & s_{2} s_{1} s_{2} s s_{2}^{-1} s_{1} s_{2}^{-1} s^{-1}=1, s s_{1}^{-1} s s_{2}^{-1} s s_{1} s s_{2}=1, \\
& \left.s_{1} s_{2} s_{1} s^{-1} s_{1}^{-1} s_{2} s_{1}^{-1} s=1\right\rangle
\end{aligned}
$$

is geometric, that is, it arises from the genus 3 Heegaard diagram of $M_{2}$ depicted in Figure 2. The abelianization of $G_{2}$ is isomorphic to $\mathbb{Z}_{2} \oplus \mathbb{Z}_{2} \oplus \mathbb{Z}_{4}$. In particular, the Heegaard genus of $M_{2}$ is 3. The volume of $M_{2}$ is equal to $(0,2222292(166) \cdots) \cdot 24 \sim 5,3335$.

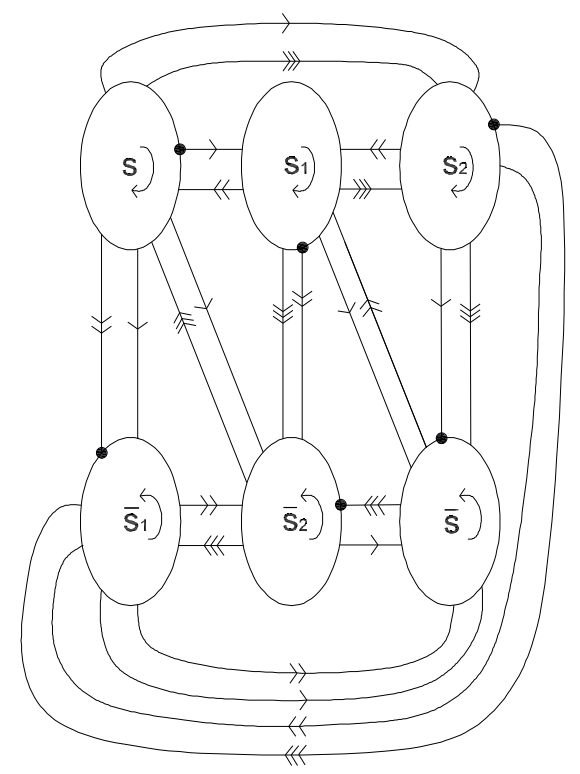

Figure 2. A minimal Heegaard diagram of $M_{2}=\mathbb{H}^{3} / G_{2}$ 


\section{A non-compact non-orientable hyperbolic space form of finite volume with two generators}

The starting Coxeter group $C$ will have such a fundamental tetrahedron $A_{0} A_{1} A_{2} A_{3}$ having the vertices $A_{0}$ and $A_{3}$ at the absolute of $\mathbb{H}^{3}$. This fact can be read off from the Coxeter diagram in Figure 3 (for more details, see e.g. [24]). Namely, the "stabilizer" $C_{0}=\left\langle m_{1}, m_{2}, m_{3}\right\rangle$ of $A_{0}$ (and that of $\left.A_{3}\right)$ is the Euclidean Coxeter group $\mathbf{p} 4 \mathbf{m}=* 244$ in the usual denotations. Introducing the half-turn $r$ with axis $A_{03} A_{12}$, indicated in Figure 3 , we get a supergroup $D=\langle C, r\rangle$ again. Bisecting $A_{0} A_{1} A_{2} A_{3}$ at the edge $A_{0} A_{3}$, we have a fundamental domain $\mathcal{F}_{D}$ which contains the vertex $A_{1}$. The stabilizer $C_{1}=\left\langle m_{0}, m_{2}, m_{3}\right\rangle$ of $A_{1}$ in $D$ is again a Coxeter point group. We unite the $C_{1}$ images of $\mathcal{F}_{D}$ to get a concave polyhedron $\mathbb{P}_{G}$ for the group $G$ to be constructed (see Figure 3). Now $\mathbb{P}_{G}$ has four broken faces (one of them is indicated more clearly in the upper-left diagram of Figure 3), two "ordinary" vertices (i.e., the $C_{1}$-images of $A_{0}$ ) and four "additional" vertices (i.e., the $C_{1}$-images of $A_{3}$ ). All vertices of $\mathbb{P}_{G}$ are ends (at the absolute) and will be equivalent under $G$. They form a cusp. The (unique!) fixed point free identifications of $\mathbb{P}_{G}$ are generated by screw motions $s: S \rightarrow \bar{S}$ and a glide reflection $t: T \rightarrow \bar{T}$ indicated in Figure 3 by corresponding arrows. Here $S, \bar{S}, T$ and $\bar{T}$ are the faces of $\mathbb{P}_{G}$ which are to be glued in pairs. We must verify, as before, that the proper points of the identified polyhedron $\mathbb{P}_{G}$ all have ball-like neighbourhoods. At the same time, we see that $G$ acts freely. According to the one edge class, the fundamental group $G$ has one defining relation. The space form $M=\mathbb{H}^{3} / G$ is non-orientable because of the generating glide reflection $t$. In fact, $t$ is an orientation reversing isometry. Summarizing, we have:

Theorem 4.1. With the above notation, the space form $M=\mathbb{H}^{3} / G$ is a noncompact non-orientable hyperbolic 3-manifold with one cusp and finite volume $1,8319311884 \cdots=2 \times($ Catalan's constant). The fundamental group $G$ has the 2-generator presentation

$$
G=\left\langle s, t: s t s^{-1} t^{-1} s t^{-1} s t=1\right\rangle
$$

by the Poincaré algorithm which corresponds to a spine of the manifold (that is, a 2-dimensional cell complex with just one 0-cell such that the manifold collapses onto it). The abelianization of $G$ is isomorphic to $\mathbb{Z}_{2} \oplus \mathbb{Z}$.

By construction, the space form $M=\mathbb{H}^{3} / G$ is precisely the unique nonorientable 1-cusped 3-manifold made from two ideal tetrahedra in the census listed in [4]. Indeed, $M$ is precisely the manifold $N 2_{1}$ from [4]. In particular, the isometry group of $M$ is isomorphic to $\mathbb{Z}_{2}$. It is known that the Gieseking manifold is the only nonorientable 1-cusped hyperbolic 3-manifold realizing the minimum volume (which is $1,0149416064 \cdots$ ). For the proof, see [1]. This manifold is obtained by identifying the faces of a single ideal tetrahedron; it has the first integral homology group and the isometry group both isomorphic to $\mathbb{Z}_{2}$. Now applying Agol's result [2], we have: 

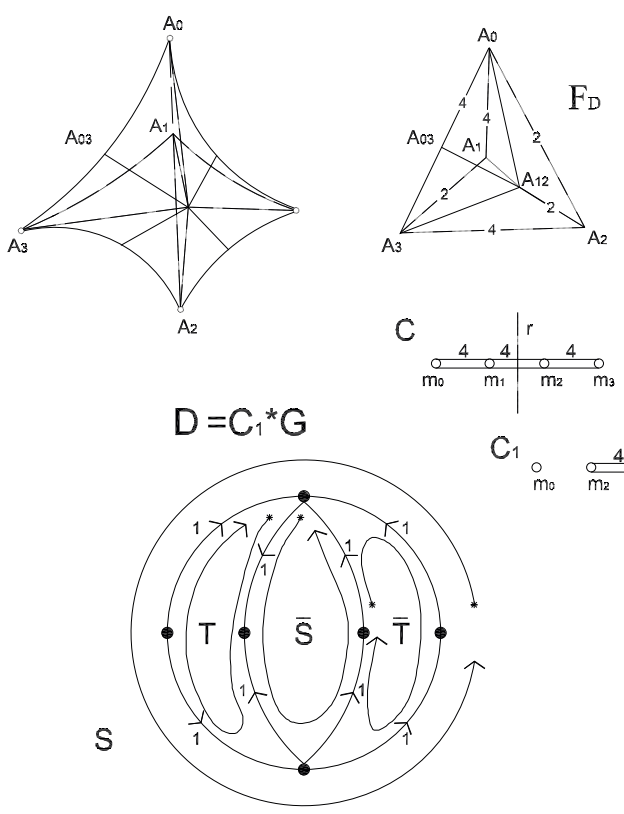

$\mathrm{C}_{1} \stackrel{4}{\stackrel{4}{\mathrm{~m}_{0} \quad \mathrm{~m}_{3}}}$

Figure 3. The hyperbolic manifold $M=\mathbb{H}^{3} / G$

Theorem 4.2. The space form $M=\mathbb{H}^{3} / G$ constructed above is the second smallest volume nonorientable hyperbolic 3-manifold with one cusp. Furthermore, the (hyperbolic) 2-fold unbranched coverings of $M$ are isometric to the Whitehead link complement and the $(-2,3,8)$-pretzel link complement in the 3-sphere.

Proof. The 2-fold unbranched coverings of $M$ correspond to different epimorphisms of $H_{1}\left(M ; \mathbb{Z}_{2}\right) \cong \mathbb{Z}_{2} \oplus \mathbb{Z}_{2}$ onto $\mathbb{Z}_{2}$. These manifolds are orientable hyperbolic with two cusps, and their volume is equal to $3,6638623767 \cdots=4 \times$ (Catalan's constant). So they are homeomorphic to the Whitehead link complement and the $(-2,3,8)$-pretzel link complement in the 3-sphere, as very recently proved by Agol in [2], Theorem 3.6.

Finally, we observe that the one-relator group $G$ in Theorem 4.1 is properly 3-realizable in the sense of [5], i.e., there exists a compact 2-polyhedron $K$ with $\pi_{1}(K) \cong G$ whose universal cover $\widetilde{K}$ is proper homotopy equivalent to a 3-manifold. In our case, $K$ is a spine of the space form $M$ (for example, the spine which corresponds to the finite presentation of $G$ ) and $\widetilde{K}$ is proper homotopy equivalent to $\mathbb{H}^{3}$. 

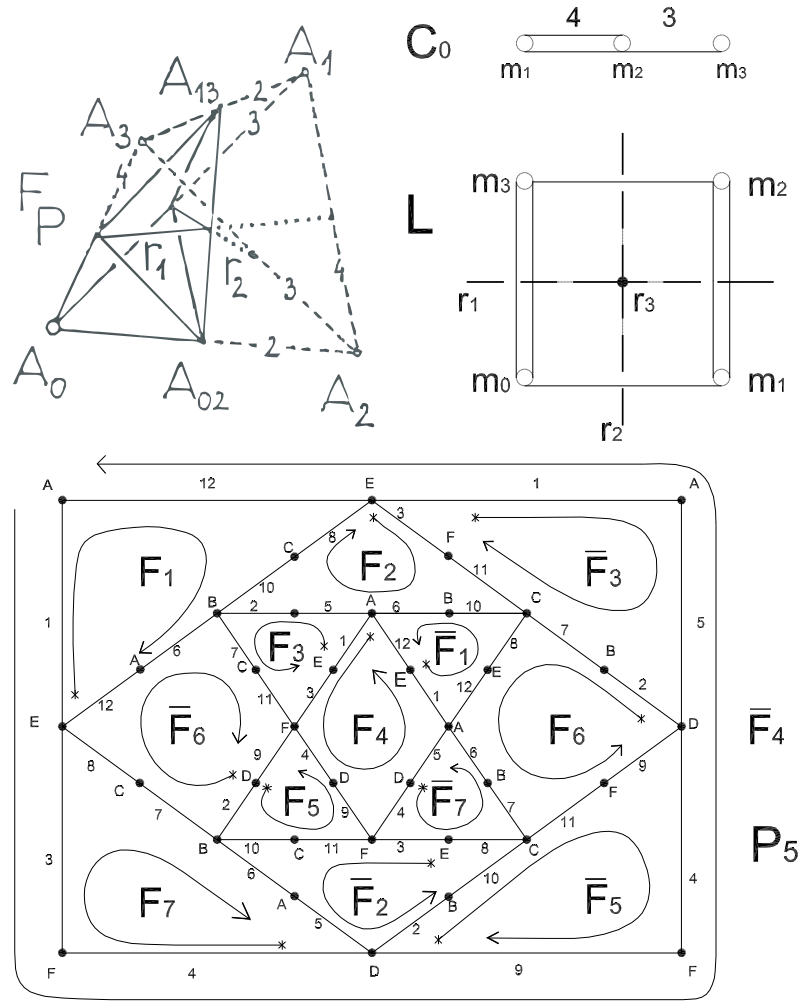

Figure 4 . The hyperbolic manifold $M_{5}=\mathbb{H}^{3} / G_{5}$

\section{Two hyperbolic Archimedian space forms with 2-generated fundamental groups}

Consider the Lanner group $L$ again (see Figure 4). We introduce the halfturn changing the mirrors $m_{0}$ and $m_{1}$ to $m_{3}$ and $m_{2}$, respectively, as in Section 2 , but now denote it by $r_{1}$. We also introduce the half-turn $r_{2}$ changing $m_{0}$ and $m_{2}$ to $m_{1}$ and $m_{3}$, respectively. This means that we extend $L$ by the dihedral group 222 to get the supergroup $P=\langle 222, L\rangle$ as a semidirect product. To get an appropriate fundamental domain $\mathcal{F}_{P}$ for $\mathrm{P}$, we put two perpendicular planes through the third half-turn axis: the first plane contains the axis of $r_{1}$ and the second contains the axis of $r_{2}$. These planes cut off $A_{0} A_{1} A_{2} A_{3}$, the domain $\mathcal{F}_{P}$, say, with vertex $A_{0}$. The stabilizer $C_{0}$ of $A_{0}$ in $P$ is $\left\langle m_{1}, m_{2}, m_{3}\right\rangle$ again. We unite the $C_{0}$-images of $\mathcal{F}_{P}$ in order to get a new concave fundamental polyhedron, denoted by $\mathbb{P}_{5}$ in Figure 4 , for the group $G_{5}$ to be constructed. Combinatorially, $\mathbb{P}_{5}$ becomes the well-known Archimedian solid of symbol (3 
434 ). Its "ordinary" vertices are the $C_{0}$-images of the midpoint $A_{02}$ and the "additional" ones are the $C_{0}$-images of $A_{13}$. In Figure 4 , the generating screw motions for the group $G_{5}$ are described also by arrows. For the free action of $G_{5}$ on $\mathbb{H}^{3}$, we have to guarantee the ball-like neighbourhoods for each point of the identified polyhedron arising from $\mathbb{P}_{5}$.

Let us describe the situation:

1) We have good joins as in the midpoints of the corresponding "tetragonal" faces of $\mathbb{P}_{5}$ (these are the $C_{0}$-images of $A_{03}$ ) as in the midpoints of the associated "trigonal" faces of $\mathbb{P}_{5}$ (which are the $C_{0}$-images of $A_{01}$ ). Namely, the "ordinary" vertices are mapped onto the "additional" ones and vice versa for each generator. This important argument was not stated previously for brevity. Moreover, the argument on the number of the $C_{0}$-images of $\mathcal{F}_{P}$, say at $A_{03}$ and $A_{01}$, as in Section 2 also hold in this case.

2) Each edge equivalence class consists of four edges of $\mathbb{P}_{5}$, indeed. This is needed because rectangles occur at them in $\mathbb{P}_{5}$. Let $s_{i}, i=1, \ldots, 7$, denote the isometry which identifies the faces $F_{i}$ with $\bar{F}_{i}$ according to the arrows depicted in Figure 4. We enumerate the corresponding cycle relations.

3) There are six vertex equivalence classes, which are indicated in Figure 4 by $A, B, \ldots, F$. Each of them consists of two "ordinary" vertices and four "additional" ones, forming a whole ball-like neighbourhood as a consequence of the conditions above.

\begin{tabular}{|c|c|}
\hline label of the edge & relation \\
\hline \hline 1 & $s_{4} s_{1} s_{4} s_{3}^{-1}=1$ \\
\hline 2 & $s_{6} s_{5} s_{2}^{-1} s_{3}=1$ \\
\hline 3 & $s_{2} s_{7}^{-1} s_{4}^{-1} s_{3}=1$ \\
\hline 4 & $s_{7} s_{4} s_{5}^{-1} s_{4}=1$ \\
\hline 5 & $s_{7} s_{4} s_{3}^{-1} s_{2}=1$ \\
\hline 6 & $s_{7} s_{6} s_{1} s_{2}=1$ \\
\hline 7 & $s_{7} s_{6} s_{3} s_{6}=1$ \\
\hline 8 & $s_{6} s_{7} s_{2}^{-1} s_{1}=1$ \\
\hline 9 & $s_{4} s_{5}^{-1} s_{6}^{-1} s_{5}^{-1}=1$ \\
\hline 10 & $s_{5} s_{2}^{-1} s_{1} s_{2}=1$ \\
\hline 11 & $s_{5} s_{6} s_{3} s_{2}=1$ \\
\hline 12 & $s_{4} s_{1} s_{6} s_{1}=1$ \\
\hline
\end{tabular}

Summarizing, the supergroup $P$ is decomposed in the form $P=C_{0} * G_{5}$, with $G_{5}$ acting freely on $\mathbb{H}^{3}$. Let $M_{5}$ denote the closed hyperbolic 3 -manifold $\mathbb{H}^{3} / G_{5}$. What is more important is that from the relations before, we can express the generators $s_{2}, s_{3}, s_{5}, s_{6}$ and $s_{7}$ in terms of $s_{1}$ and $s_{4}$. In fact, we have:

(1) $s_{3}=s_{4} s_{1} s_{4}$,

(12) $s_{6}=s_{1}^{-1} s_{4}^{-1} s_{1}^{-1}$,

(7) $s_{7}=s_{6}^{-1} s_{3}^{-1} s_{6}^{-1}=s_{1} s_{4} s_{1} s_{4}^{-1} s_{1}^{-1} s_{4}^{-1} s_{1} s_{4} s_{1}$, 
(8) $s_{2}=s_{1} s_{6} s_{7}=s_{1} s_{4}^{-1} s_{1}^{-1} s_{4}^{-1} s_{1} s_{4} s_{1}$,

(2) $s_{5}=s_{6}^{-1} s_{3}^{-1} s_{2}=s_{1} s_{4} s_{1} s_{4}^{-1} s_{1}^{-1} s_{4}^{-1} s_{1} s_{4}^{-1} s_{1}^{-1} s_{4}^{-1} s_{1} s_{4} s_{1}$.

So the fundamental group $G_{5}$ of $M_{5}$ is generated by $s_{1}$ and $s_{4}$. Substituting the above formulae into the relations, we obtain that Relation (3) is an identity, Relations (4) and (9) are equivalent to the relation

$$
\left(s_{1} s_{4}\right)^{3} s_{1} s_{4}^{-1} s_{1}^{-1} s_{4}^{-1}\left(s_{1} s_{4}\right)^{2} s_{1}^{-1} s_{4}^{-1} s_{1}^{-1}\left(s_{4} s_{1}\right)^{2} s_{4}^{-1} s_{1}^{-1} s_{4}^{-1}=1,
$$

and Relations (5), (6), (10) and (11) are equivalent to the relation

$$
s_{1}^{2} s_{4}^{-1} s_{1}^{-1} s_{4}^{-1} s_{1} s_{4} s_{1}^{2} s_{4} s_{1} s_{4}^{-1} s_{1}^{-1} s_{4}^{-1}=1 .
$$

From the relations, we also get

$$
s_{2}=s_{1} s_{4}^{-1} s_{1}^{-1} s_{4}^{-1} s_{1} s_{4} s_{1}=s_{1}^{-1} s_{4} s_{1} s_{4} s_{1}^{-1} s_{4}^{-1} s_{1}^{-1} .
$$

Then $G_{5}$ admits a 2-generator 2-relator presentation. Of course, the other two generators, and no less, must also be taken into account. In fact, the abelianization of $G_{5}$ is isomorphic to $\mathbb{Z}_{2} \oplus \mathbb{Z}_{8}$.

Theorem 5.1. The fundamental group $G_{5}$ of the closed connected hyperbolic 3-manifold $M_{5}=\mathbb{H}^{3} / G_{5}$ has the balanced presentation

$$
\begin{aligned}
G_{5}=\left\langle s_{1}, s_{4}:\right. & s_{1}^{2} s_{4}^{-1} s_{1}^{-1} s_{4}^{-1} s_{1} s_{4} s_{1}^{2} s_{4} s_{1} s_{4}^{-1} s_{1}^{-1} s_{4}^{-1}=1, \\
& \left.\left(s_{1} s_{4}\right)^{3} s_{1} s_{4}^{-1} s_{1}^{-1} s_{4}^{-1}\left(s_{1} s_{4}\right)^{2} s_{1}^{-1} s_{4}^{-1} s_{1}^{-1}\left(s_{4} s_{1}\right)^{2} s_{4}^{-1} s_{1}^{-1} s_{4}^{-1}=1\right\rangle .
\end{aligned}
$$

This presentation is geometric and arises from the genus 2 Heegaard diagram of $M_{5}$ depicted in Figure 5.

In Figure 6, we show the $\{4,6,8\}$ realization of the 2 -generator closed hyperbolic space form $M_{5}=\mathbb{H}^{3} / G_{5}$, constructed uniquely by the starting edge class marked with bold arrows. This is a nice polyhedron, denoted by $\mathcal{F}_{G_{5}}$.

The group $G_{5}$ is now generated by the isometries $S_{1}$ and $S$ (see Figure 6 ). The side pairing of the boundary faces of $\mathcal{F}_{G_{5}}$ is given by matching each pair of faces labelled by an isometry with its inverse. The face pairing generators are induced and expressed by $S$ and $S_{1}$ step-by-step. The three edges in each class (24 classes) bound $4-$ and 6-, 6- and 8-, and 8- and 4-gons, guaranteeing the ball-like neighbourhood to each point.

For the fundamental group of $M_{5}$, we get a new geometric presentation with generators $S_{1}$ and $S$ and relations:

$$
S_{1}^{3} S^{-2}\left(S_{1} S\right)^{2} S_{1} S^{-2}=1
$$

and

$$
S_{1} S S_{1} S^{-2} S_{1}^{-1} S^{2} S_{1}^{-1} S^{-3} S_{1}^{-1} S^{2} S_{1}^{-1} S^{-2}=1 .
$$

Setting $S^{-1}=s_{1} s_{4}$ and $S_{1}^{-1}=s_{1}$ (with inverse relation $s_{4}=S_{1} S^{-1}$ ), the first relation above becomes the inverse of the first relation in the statement of Theorem 5.1, while the second relation above becomes (up to cyclic permutation) the second relation in the statement of Theorem 5.1. Let us turn to the starting motivation. If we take the presentation of $G_{2}$ from Section 2 (Figure 1) with 


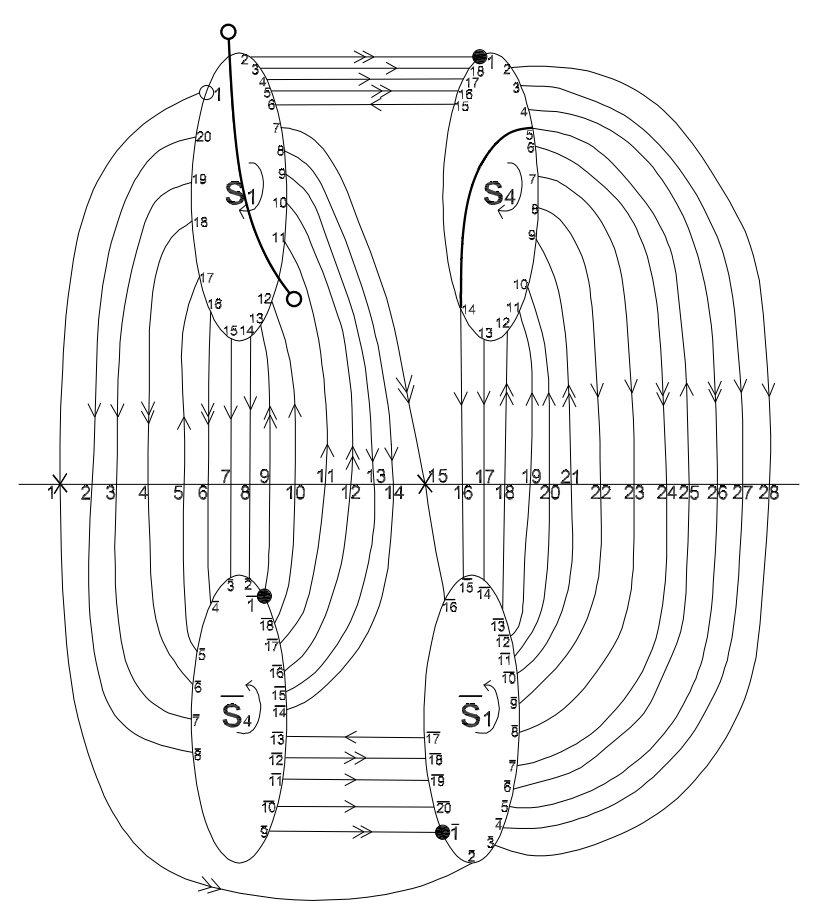

FiguRe 5. A Heegaard diagram of the hyperbolic manifold $M_{5}=\mathbb{H}^{3} / G_{5}$

generators denoted now with primes, $s^{\prime}, s_{1}^{\prime}$ and $s_{2}^{\prime}$, and the presentation of $G_{5}$ as before, then setting

$$
\begin{gathered}
s^{\prime}=s_{4}, \\
s_{1}^{\prime}=s_{6}=s_{1}^{-1} s_{4}^{-1} s_{1}^{-1}, \\
s_{2}^{\prime}=s_{2}^{-1}=s_{1}^{-1} s_{4}^{-1} s_{1}^{-1} s_{4} s_{1} s_{4} s_{1}^{-1}=s_{1} s_{4} s_{1} s_{4}^{-1} s_{1}^{-1} s_{4}^{-1} s_{1},
\end{gathered}
$$

we obtain $G_{2} \triangleleft G_{5}$. Geometrically, this is clear if we study the corresponding pairings of faces on the boundaries of the polyhedra $\mathbb{P}_{2}$ and $\mathbb{P}_{5}$. Moreover, $G_{2}$ is of index 2 in $G_{5}$ since the supergroup $N$ of $G_{2}$ is a subgroup of index 2 in the supergroup $P$ of $G_{5}$. Furthermore, the volume of $\mathbb{P}_{2}$ is two times larger than that of $\mathbb{P}_{5}$, and hence the volume of $M_{5}$ is 2,6668. Formally, the three relations of $G_{2}$ are consequences of the two relations of $G_{5}$, which are more tedious to handle.

Theorem 5.2. The genus 3 hyperbolic closed 3-manifold $M_{2}=\mathbb{H}^{3} / G_{2}$ is the 2-fold unbranched covering of the genus 2 hyperbolic closed 3-manifold $M_{5}=$ $\mathbb{H}^{3} / G_{5}$. Furthermore, we have $\operatorname{vol}\left(M_{2}\right)=2 \operatorname{vol}\left(M_{5}\right)$, where $\operatorname{vol}\left(M_{5}\right) \sim 2,6668$. 


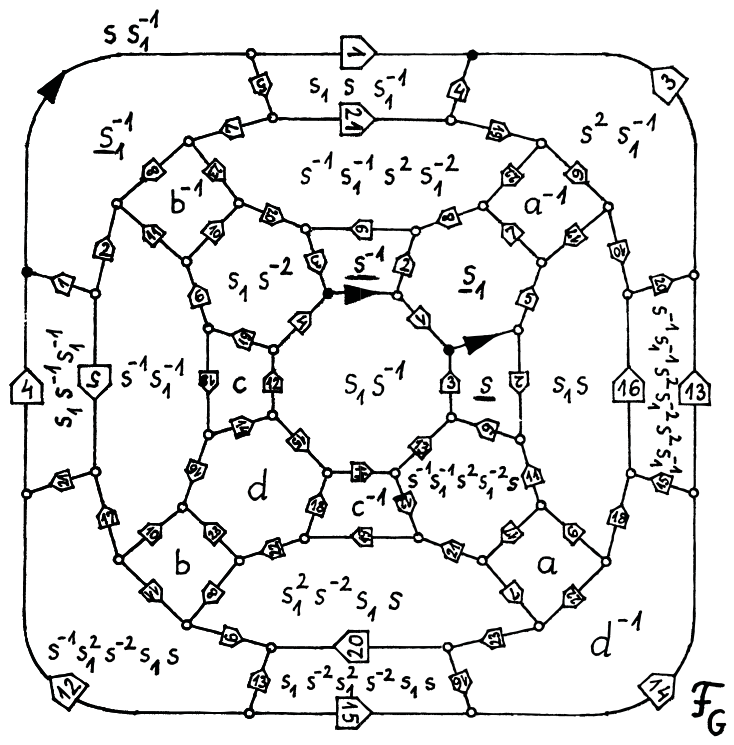

Figure 6 . The $\{4,6,8\}$ realization of the hyperbolic manifold $M_{5}$

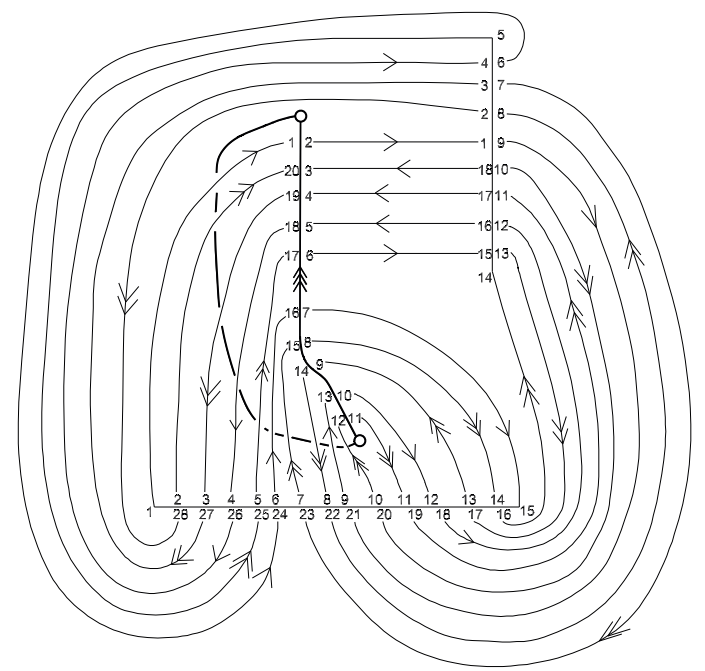

Figure 7. The 3-bridge link $\mathcal{L}$ with three components

The (extended) Heegaard diagram of genus 2, shown in Figure 5, has an orientation-preserving involution which fixes the marked axis of the ellipses $s_{1}$ 


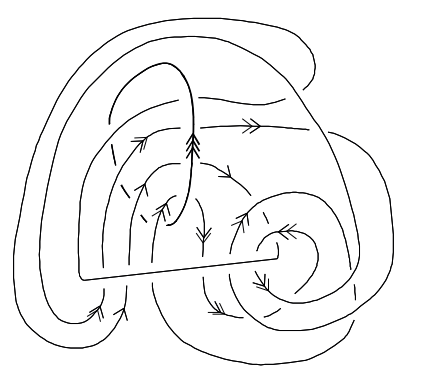

(a)

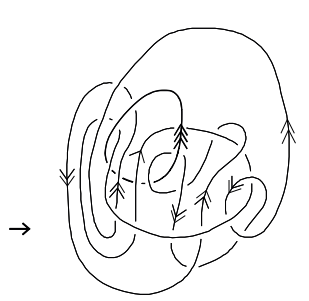

(b)

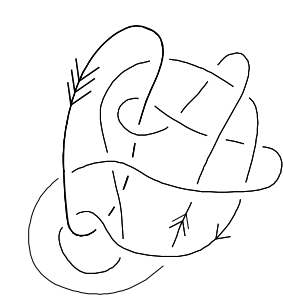

(d)

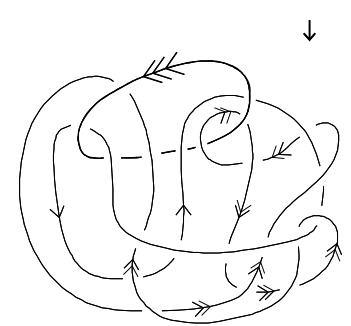

(c)

FiguRE 8. The link $\mathcal{L}$, up to Reidemeister moves

and $s_{4}$ and the axis joining the vertices 1 and 15 of the horizontal circle (closed at the infinity). Then by [3] and [21], the manifold $M_{5}$ is the 2 -fold covering of the 3 -sphere branched over the 3 -bridge link $\mathcal{L}$ with three components, drawn in Figure 7. Using the Reidemeister moves in Figure 8, we get:

Theorem 5.3. The hyperbolic closed 3-manifold $M_{5}=\mathbb{H}^{3} / G_{5}$ is the 2-fold covering of the 3-sphere branched over the 3-bridge link $\mathcal{L}$ with three trivial components, depicted in Figure $8(\mathrm{~d})$. The link $\mathcal{L}$ is chiral, $\pi$-hyperbolic and $\operatorname{vol}\left(\mathbb{S}^{3} \backslash \mathcal{L}\right)=9,503403931$. The symmetry group of $\mathcal{L}$ is isomorphic to $\mathbb{Z}_{2} \times \mathbb{Z}_{2}$, and the isometry group of $M_{5}$ is $\mathbb{Z}_{2} \times \mathbb{Z}_{2} \times \mathbb{Z}_{2}$.

Proof. Let $\tau$ denote the involution of $M_{5}$ such that $M_{5} /\langle\tau\rangle$ is topologically the 3 -sphere with branch set the 3 -bridge link $\mathcal{L}$ with branching index 2 on its components. In the language of orbifolds, the quotient $M_{5} /\langle\tau\rangle$ is a hyperbolic 3 -orbifold $\mathcal{O}(\mathcal{L})$ whose underlying topological space is the 3 -sphere and whose singular set is $\mathcal{L}$ with singular index 2 .

The symmetry group $\operatorname{Sym}\left(\mathbb{S}^{3}, \mathcal{L}\right)$ of $\mathcal{L}$, which is isomorphic to $\operatorname{Iso} \mathcal{O}(\mathcal{L})$, is $\mathbb{Z}_{2} \times \mathbb{Z}_{2}$ by SnapPea. Since $\mathcal{L}$ has three components, Case 1 in Theorem 1 of [20] occurs (see also the proof of the theorem on p. 82), i.e., $\tau$ is central in Iso $M_{5}$. So we have the exact sequence

$$
1 \longrightarrow\langle\tau\rangle \cong \mathbb{Z}_{2} \longrightarrow \text { Iso } M_{5} \longrightarrow \text { Iso } \mathcal{O}(\mathcal{L})=\mathbb{Z}_{2} \times \mathbb{Z}_{2} \longrightarrow 1,
$$


which implies the desired result.

Remark. Since each component $K_{i}$ of $\mathcal{L}$ is trivial, the $\mathbb{Z}_{2}$-branched covering $N_{i}$ of $\mathcal{L}$ along $K_{i}$ is the 3 -sphere for each $i=1,2,3$. Let $R_{i j}$ be the 2 -fold covering of $M_{k} \cong \mathbb{S}^{3}$ branched over the union of the preimages $\widetilde{K}_{i}, \widetilde{K}_{j}$ of $K_{i}, K_{j}$ for $i \neq j \neq k, i, j, k \in\{1,2,3\}$. Then $R_{i j}$ is a closed hyperbolic 3 -manifold of genus 2. Let $M$ denote the $\left(\mathbb{Z}_{2} \times \mathbb{Z}_{2} \times \mathbb{Z}_{2}\right)$-branched covering of $\mathcal{L}$. Then $M$ is a $\mathbb{Z}_{2}$-covering of $R_{12}, R_{13}$ and $R_{23}$ and a $\left(\mathbb{Z}_{2} \times \mathbb{Z}_{2}\right)$-covering of $N_{i} \cong \mathbb{S}^{3}$ for $i=1,2,3$. Hence $M$ is hyperbolic and hyperelliptic.

It is proved in [15] that the two-fold unbranched coverings of genus two 3-manifolds are hyperelliptic. As a consequence, we have:

Corollary 5.4. The closed hyperbolic 3-manifold $M_{2}=\mathbb{H}^{3} / G_{2}$ is hyperelliptic. The isometry group of $M_{2}$ is isomorphic to $\mathbb{Z}_{2} \times \mathbb{Z}_{2} \times \mathbb{Z}_{2} \times \mathbb{Z}_{2}$.

The manifolds $M_{2}$ and $M_{5}$ are missing in the Weeks census of closed hyperbolic 3-manifolds [25]. The reason is that Weeks did not pose himself the problem of listing the closed hyperbolic 3-manifolds and volumes without gaps. He intentionally neglected some manifolds whose geometries were close to those of the corresponding cusped manifolds.

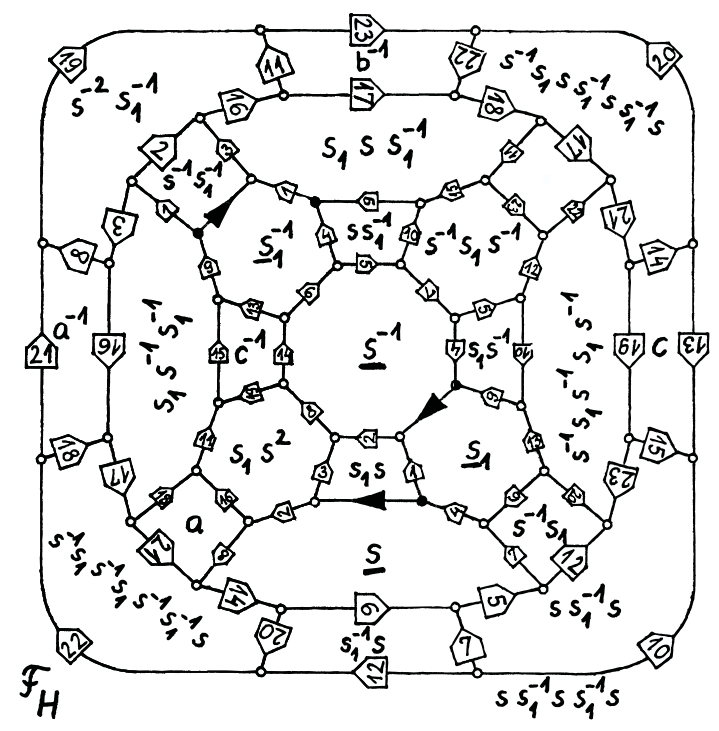

FiguRE 9 . The $\{4,6,8\}$ realization of the hyperbolic manifold $M^{\prime}=\mathbb{H}^{3} / H$

In Figure 9, we show the $\{4,6,8\}$ realization of the 2 -generator closed hyperbolic space form $M^{\prime}=\mathbb{H}^{3} / H$, constructed uniquely by the starting edge 
class marked with bold arrows. The other face pairing generators are induced and expressed by $S$ and $S_{1}$ step-by-step. It is only a conjecture that the other 2-generator 3-space form from the Archimedian solid $\{8,6,4\}$ does not exist. The fundamental group $H \cong \pi_{1}\left(M^{\prime}\right)$ has a balanced presentation with generators $S_{1}$ and $S$ and relations

$$
S_{1} S S_{1}^{-1} S S_{1}^{-1} S^{2}\left(S_{1}^{-1} S\right)^{2} S_{1} S^{2}=1
$$

and

$$
S_{1} S S_{1}^{-1} S^{-1}\left(S_{1} S^{-1}\right)^{2} S_{1}^{-1} S S_{1} S^{3}=1
$$

The abelianization of $H$ is isomorphic to $\mathbb{Z}_{2} \oplus \mathbb{Z}_{10}$. The above presentation is geometric and arises from the genus 2 Heegaard diagram of $M^{\prime}$ depicted in Figure 10.

In particular, $M^{\prime}$ has Heegaard genus 2. This diagram has an orientationpreserving involution which fixes the marked axis of the ellipses labelled $S_{1}$ and $S$ and the axis joining the vertices 2 and 9 of the horizontal circle (closed at the infinity). Then by [3] and [21], the manifold $M^{\prime}$ is the 2-fold covering of the 3 -sphere branched over the 3 -bridge link $\mathcal{L}^{\prime}$ with three trivial components, drawn in Figure 11. Following a similar reasoning as the proof of Theorem 5.3, we obtain the following result:

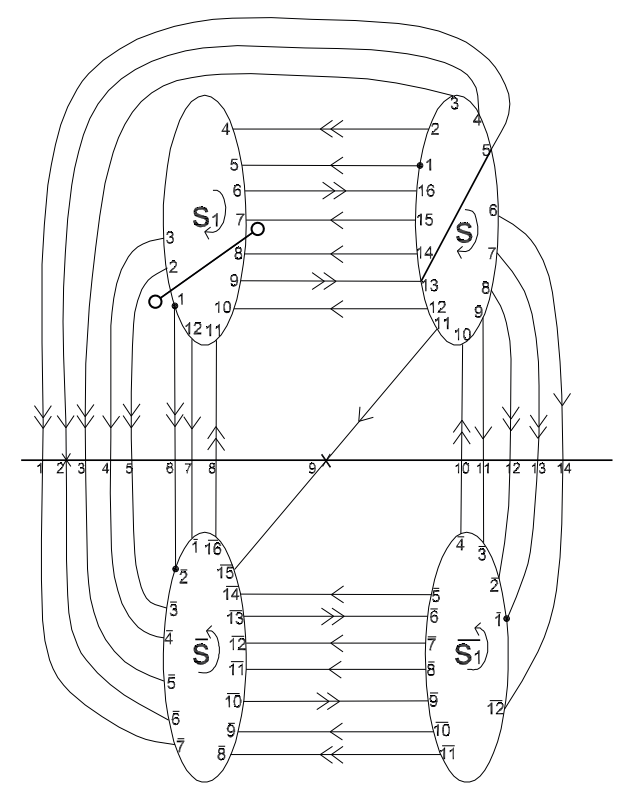

Figure 10. A Heegaard diagram of the hyperbolic manifold $M^{\prime}=\mathbb{H}^{3} / H$ 


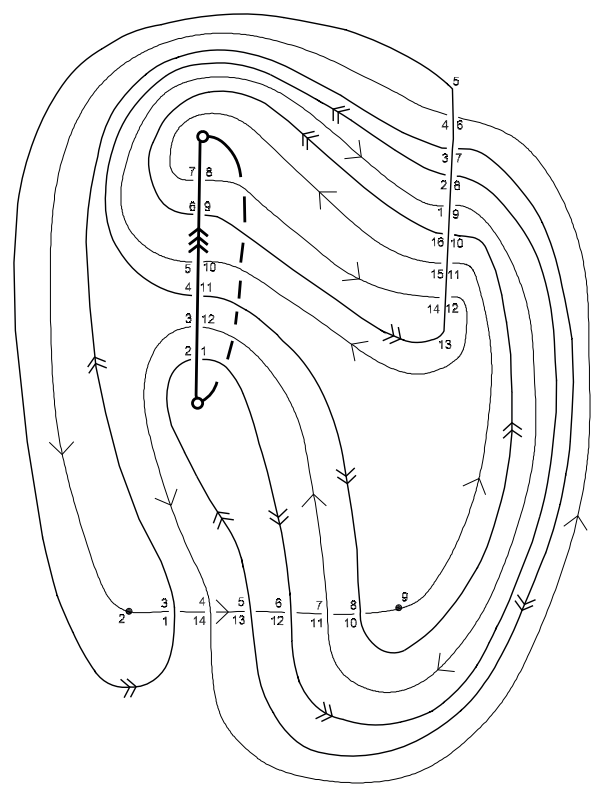

Figure 11. The 3 -bridge link $\mathcal{L}^{\prime}$ with three components

Theorem 5.5. The hyperbolic closed 3-manifold $M^{\prime}=\mathbb{H}^{3} / H$ is the 2-fold covering of the 3-sphere branched over the 3-bridge link $\mathcal{L}^{\prime}$ with three trivial components, depicted in Figure 11. The link $\mathcal{L}^{\prime}$ is chiral, $\pi$-hyperbolic and $\operatorname{vol}\left(\mathbb{S}^{3} \backslash \mathcal{L}^{\prime}\right)=10,2758824$. The symmetry group of $\mathcal{L}^{\prime}$ is isomorphic to $\mathbb{Z}_{2}$, and the isometry group of $M^{\prime}$ is $\mathbb{Z}_{2} \times \mathbb{Z}_{2}$.

Finally, note that the volume of the closed hyperbolic 3-manifold $M^{\prime}=$ $\mathbb{H}^{3} / H$ equals that of $M_{5}=\mathbb{H}^{3} / G_{5}$ but that they are not homeomorphic because they have different first integral homology groups.

Acknowledgements. Work performed under the auspices of the section G.N.S.A.G.A. of the C.N.R. (National Research Council) of Italy and partially supported by the Ministero per la Ricerca scientifica e Tecnologica of Italy within the project Proprietá Geometriche delle Varietá Reali e Complesse, and by the Slovenian-Hungarian Intergovernmental Cooperation Programme. The third author is supported by a grant from L'Oreal Italy within the project "Women for Science". Many thanks are due to the referee for his/her suggestions and useful remarks which were most valuable in preparing the last version of the paper. 


\section{References}

[1] C. Adams, The noncompact hyperbolic 3-manifold of minimal volume, Proc. Amer. Math. Soc. 100 (1987), no. 4, 601-606.

[2] I. Agol, The minimal volume orientable hyperbolic 2-cusped 3-manifolds, Proc. Amer. Math. Soc. 138 (2010), no. 10, 3723-3732.

[3] J. S. Birman and H. M. Hilden, Heegaard splittings of branched coverings of $\mathbb{S}^{3}$, Trans. Amer. Math. Soc. 213 (1975), 315-352.

[4] P. J. Callahan, M. V. Hildebrand, and J. R. Weeks, A census of cusped hyperbolic 3-manifolds, Math. Comp. 68 (1999), no. 225, 321-332.

[5] M. Càrdenas, F. F. Lasheras, A. Quintero, and D. Repovš, One-relator groups and proper 3-realizability, Rev. Mat. Iberoam. 25 (2009), no. 2, 739-756.

[6] H. S. M. Coxeter and W. O. J. Moser, Generators and Relations for Discrete Groups, 4th edition, Ergeb. der Math., Neue Folge Ed., 14, Springer Verlag, Berlin-New York, 1960.

[7] H. M. Farkas and I. Kra, Riemann Surfaces, Springer Verlag, Berlin, 1992.

[8] A. T. Fomenko and S. V. Matveev, Algorithmic and Computer Methods for ThreeManifolds, Math. and Its Appl. 425 Kluwer Acad. Publ., Dordrecht-Boston-London, 1997.

[9] H. Helling, A. C. Kim, and J. L. Mennicke, Some honey-combs in hyperbolic 3-space, Comm. Algebra 23 (1995), no. 14, 5169-5206.

[10] - A geometric study of Fibonacci groups, J. Lie Theory 8 (1998), no. 1, 1-23.

[11] F. Lanner, On complexes with transitive groups of automorphisms, Lunds Univ. Math. Sem. 11 (1950), 1-71.

[12] V. S. Makarov, Geometric methods for constructing discrete isometry groups of Lobaschevskian space, Viniti, Itogi Nauki i Techniki, Problemy Geometrii 15 (1983), 3-59 (In Russian).

[13] B. Maskit, On Poincaré's theorem for fundamental polygons, Advances in Math. 7 (1971), 219-230.

[14] A. D. Mednykh, Three-dimensional hyperelliptic manifolds, Annals of Global Analysis and Geometry 8 (1990), 13-19.

[15] A. Mednykh and M. Reni, Twofold unbranched coverings of genus two 3-manifolds are hyperelliptic, Israel J. Math. 123 (2001), 149-155.

[16] E. Molnár, Space forms and fundamental polyhedra, Proceedings of the conference on differential geometry and its applications, Part 1 (Noé Město na Moravě, 1983), 91-103, Charles Univ., Prague, 1984.

[17] - Minimal presentation of the 10 compact Euclidean space forms by fundamental domains, Studia Sci. Math. Hungar. 22 (1987), no. 1-4, 19-51.

[18] _ Two hyperbolic football manifolds, Differential geometry and its applications (Dubrovnik, 1988), 217-241, Univ. Novi Sad, Novi Sad, 1989.

[19] , Polyhedron complexes with simply transitive group actions and their realizations, Acta Math. Hungar. 59 (1992), no. 1-2, 175-216.

[20] M. Reni, The isometry groups of genus 2 hyperbolic 3-manifolds, Kobe J. Math. 15 (1998), no. 1, 77-84.

[21] M. Takahashi, An alternative proof of Birman- Hilden- Viro's theorem, Tsukuba J. Math. 2 (1978), 27-34.

[22] W. P. Thurston, Three-Dimensional Geometry and Topology, (S. Levy ed.), Princeton Univ. Press, Princeton, N.J., 1997.

[23] A. Yu. Vesnin and A. D. Mednykh, Three-dimensional hyperbolic manifolds of small volume with three hyperelliptic involutions, Siberian Math. Journal 40 (1999), no. 5, 873-886.

[24] E. B. Vinberg and O. V. Shvartsman, Discrete groups of motions of spaces of constant curvature, Geometry, II, 139-248, Encyclopaedia Math. Sci., 29, Springer, Berlin, 1993. 
[25] J. Weeks, SnapPea: A computer program for creating and studying hyperbolic 3manifolds, www.geometrygames.org.

[26] J. A. Wolf, Spaces of Constant Curvature, Univ. of California, Berkeley, California, 1972; Russian translation: Izd. "Nauka", Moskow, 1982.

\section{Alberto Cavicchioli}

Dipartimento di Matematica

Università di Modena e RegGio E.

Via Campi 213/B, 41100 Modena, Italy

E-mail address: alberto.cavicchioli@unimore.it

EMIL MOLnÁR

Department of Geometry

Institute OF Mathematics

BudAPEST University of TeChNOLOGy AND ECONOMiCs

Budapest Xi, Egry J. U. 1, H. II. 22, H-1521, Budapest, Hungary

E-mail address: emolnar@math.bme.hu

Agnese I. Telloni

Dipartimento di MATEMATICA

Università di Modena e RegGio E.

Via Campi 213/B, 41100 Modena, Italy

E-mail address: agneseilaria.telloni@unimore.it 